\title{
Establishment reality vs. maintenance reality: how real is real enough?
}

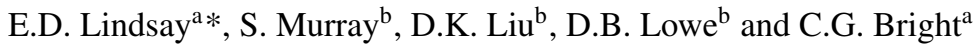 \\ ${ }^{a}$ Department of Mechanical Engineering, Curtin University, Perth, Australia $;{ }^{b}$ Faculty of Engineering, \\ University of Technology, Sydney, Australia
}

(Received 6 January 2009; final version received 13 March 2009)

\begin{abstract}
Remote and virtual laboratories are increasingly prevalent alternatives to the face-to-face laboratory experience; however, the question of their learning outcomes is yet to be fully investigated. There are many presumptions regarding the effectiveness of these approaches; foremost amongst these assumptions is that the experience must be 'real' to be effective. Embedding reality into a remote or virtual laboratory can be an expensive and time-consuming task. Significant efforts have been expended to create 3D VRML models of laboratory equipment, allowing students to pan, zoom and tilt their perspective as they see fit. Multiple camera angles have been embedded into remote interfaces to provide an increased sense of 'real-ness'. This paper draws upon the literature in the field to show that the necessary threshold for reality varies depending upon how the students are interacting with the equipment. There is one threshold for when they first interact - the establishment reality - which allows the students to familiarise themselves with the laboratory equipment, and to build their mental model of the experience. There is, however, a second, lower, threshold - the maintenance reality - that is necessary for the students' ongoing operation of the equipment. Students' usage patterns rely upon a limited subset of the available functionality, focusing upon only some aspects of the reality that has been originally established. The two threshold model presented in this paper provides a new insight for the development of virtual laboratories in the future.
\end{abstract}

Keywords: virtual laboratory; simulation; student perceptions; verisimilitude

\section{Introduction}

Remote and virtual laboratories are increasingly prevalent alternatives to the face-to-face laboratory experience; however, the question of their learning outcomes is yet to be fully investigated. There are many presumptions regarding the effectiveness of these approaches; foremost among these assumptions is that the experience must be 'real' to be effective.

The creation of a realistic simulation is not a simple process. It requires experts who understand the physical phenomena being simulated. It requires experts who can build an accurate model of these phenomena. It requires experts who can build an interface to this model. Each of these tasks requires different skills, often from different people, and as the required verisimilitude of the simulation increases, the complexity of each of these skills increases.

*Corresponding author. Email: e.lindsay@curtin.edu.au

ISSN 0304-3797 print/ISSN 1469-5898 online (C) 2009 SEFI

DOI: $10.1080 / 03043790902902906$

http://www.informaworld.com 
It is possible to achieve high degrees of realism in a simulation, but it is an expensive process. Each of the experts involved needs to be paid for, as do the computer resources used in the development of the software. For complex simulations there is further expense when they are implemented - high-end computing resources become necessary to make the software function. Some highly sophisticated simulations - such as flight simulators for pilot training - require dedicated physical infrastructure for their use, further increasing the cost.

Embedding reality into a remote or virtual laboratory can be an expensive and time-consuming task. Significant efforts have been expended to create 3D VRML models of laboratory equipment, allowing students to pan, zoom and tilt their perspective as they see fit. Multiple camera angles have be embedded into remote interfaces to provide an increased sense of 'real-ness'. There have been a wide range of impressive simulations, ranging from wind tunnels (Jia et al. 2006) to virtual refineries (Schofield et al. 2004). The investment of time, energy and money into these simulations shows that there is a clear perception that the increased realism of sophisticated simulations is worthwhile. Whether this is in fact the case bears further inspection.

\section{The effectiveness of reality}

There is a widespread presumption that a realistic simulation is a more effective learning experience. There are certainly good grounds for this presumption; however, the desire for the simulation to be realistic is in fact a simplification of a number of other objects that correlate well with realism.

Students must be able to anchor what they are learning into their prior knowledge (Ausubel 2000), much of which will have been learned in a 'real' context. Thus, a realistic experience will reduce the cognitive dissonance, and facilitate the assimilation of new knowledge. If the students have real experiences in which to anchor new knowledge, it is logical that it will be easier if these new experiences are themselves real.

Fidelity is an important aspect of establishing the presence of the simulation. Fidelity impacts on the learner's ability to transfer the knowledge they have learned. As practicing engineers, the contexts in which they will need the knowledge will be real, rather than educational simulations. Learners transfer better in high-fidelity situations, but if they do not learn in the first place, then there can be no transfer (Alessi and Trollip 1991). This is the risk of high-fidelity simulations 'increasing fidelity, which theoretically should increase transfer, may inhibit initial learning which in turn would inhibit transfer' (Alessi and Trollip 1991).

A simulation with too much fidelity - such as an aircraft cockpit with hundreds of controls and masses of feedback - can be overwhelming to a learner, particularly an inexperienced learner. Another example of where higher levels of fidelity are inappropriate was raised by Aldrich (2004), who observed that the best selling bird-watching guides use illustrations of birds rather than photographs.

There is an additional danger in the use of simulations that students lose sight of the real hardware being simulated, and instead get caught up in the 'computer game' attitude towards the software (Schofield et al. 2004). This issue - whether the students focus on the equipment being simulated, or the interface of the simulation - is known as transparency. If the simulation is transparent - and thus, by implication, more 'real' - then the students are focusing upon the real physical phenomena, rather than the artificial environment of the simulation.

A more realistic simulation offers many advantages in light of these desirable outcomes - the mental distance between the learning experience and the students' previous and future experiences is lower. In these instances reality is good, but it must not be reality for reality's sake-the 'real-ness' of the simulation must be focused to achieved the desired outcomes. 
An important tool for learning through experiments is how students deal with contradictions between their expectations and their observations. If the data they collect does not match their expectations, then they must address their discrepancy. Students can question their data, and retake measurements. Alternatively, they can question their expectations, and change their understanding of the phenomena. It is this evolution of understanding that is the objective of the laboratory exercise.

With a simulation, there is an additional third option - to question the accuracy of the simulation. Students are able to question whether the data is an accurate representation of reality, and in doing so avoid having to question their own mental models of the physical phenomena. The greater the sense of reality the students experience from the simulation, the less likely they are to fall into this trap.

The challenge is to ensure that the simulation is able to provide a level of reality that best fits the needs of the students. Too simplistic and there is a risk that the transparency will be compromised, and the simulation becomes a computer game. Too complex and there is a risk that the students will be overwhelmed with information. The challenge is further complicated by the way in which students' needs change over time.

\section{The stages of reality}

The core objective is to ensure that the level of verisimilitude matches the needs of the students as they operate the simulation. The challenge is that the needs of students vary over the course of their use of any given simulation. Their use can be broadly split into three phases - initial use, regular use and expert use.

In the initial use phase, students encounter the simulation for the first time. Everything is new to them, and they need to familiarise themselves not just with the physical phenomena being modelled, but also with the interface, the experimental procedures and a range of other potential factors. The use of standardised interfaces across a suite of simulations can help reduce the burden of learning a new interface, but ultimately this orientation is unavoidable. Students are learning in this phase, but this learning is mostly preparation for the objective-specific learning in the regular use phase.

In the regular use phase, the students have familiarised themselves with the simulation interface, developed a mental model of the physical phenomena, and are now able to explore how changing the input parameters leads to changes in the output parameters. In this way the students can address the objective-specific learning outcomes of the simulation.

In the expert use phase, the students seek to make their use of the simulation more efficient and effective. This phase is also characterised by students finding more efficient ways to implement functionality that they are already used - they seek short cuts through the simulation.

There is not a crisp distinction between the three phases, and students will move backwards and forwards through the phases. Indeed, the expert use phase will involve exploration to find new short cuts, which is a form of exploration similar to the initial use phase. The needs of the students are different in each of these phases.

A simulation provides students with a range of interaction options - they have many branches in the pathway in which they interact with the software. In the initial user phase, the students' objectives are familiarisation with the simulation, which requires them to travel through many of the branches, forwards and backwards. As they become increasingly familiar with the simulation, they will identify which of the branches they require most often, and start to identify how some of these branches link up to provide the functionality of the simulation.

In the regular use phase, the students tend to stick to the same regular pathways through the software. They have identified how to change parameters, how to collect output data, how to 
change perspectives of on-screen imagery, and now they use these skills to explore the physical phenomena. What they are doing in this process is reducing the range of pathways they take through the simulation - they are unconsciously making options in the simulation redundant.

In the expert user phase, the students consciously seek to make pathways in the software redundant. Rather than stick to their existing pathways, they look for newer, shorter pathways, and having found them, they no longer use the old approaches. Richardson et al. (2006) illustrated this clearly with their simulation of an electronics workbench, in which students could use hotkeys to snap directly to specific views of the equipment. While in the initial use phase, students were happy to pan, zoom and tilt their way across the workbench. They then quickly moved to the expert phase, where they chose specific views of the equipment, and then used hotkeys to swap between them - abandoning the pan, zoom and tilt functions completely.

Richardson's pan, zoom and tilt functions are largely redundant once students become familiar with the software, but they form a critical part of the students' initial use of the simulation. These functions are essential to help establish the reality of the simulated workbench, but are largely redundant in the maintenance of this reality.

The reality will persist as long as the transparency of the interface remains, and it is only when the simulation prevents an option to the user that the transparency will suffer. Many of the paths through the simulation are only used in the initial familiarisation stage, and as such they are only necessary in that phase. Once the students become familiar with the simulation, they narrow down the range of pathways that they use - potentially allowing for these pathways to be removed without the students noticing, and thus without a loss of transparency or reality.

\section{Different reality thresholds}

Students have different learning objectives in the different phases of their use, and these manifest in different usage patterns as they become more familiar with the simulation. Indeed, sometimes students will deliberately compromise the reality of the simulation as part of their expert user phase. This was observed by Koretsky et al. (2008) with their virtual silicon wafer factory, in which students must set the parameters for the silicon deposit process, and then test the wafers that are produced. The virtual factory contained separate interfaces for the two operations, with one interface that controlled the virtual factory, and a second that controlled the virtual testing rig. The first interface would generate experimental data and save it to disc, which would then be available through the interface to the testing rig.

The students realised that although the factory interface was displaying the 'process underway' message, representing the time taken for the manufacturing process to occur, the experimental data was in fact already saved to disc, and available at the testing station. This allowed for students to enter the production parameters, commence the process, then collect the results before the simulation had in fact 'completed' the process. This is a quicker way to access the data, but it undermines the 'reality' of the experience.

The question then becomes which is more important? Is it appropriate to make students wait for data to reinforce the concept that industrial processes are not instantaneous? Or is it better to disregard this aspect of the reality in order to allow them to run more combinations of the parameters, and understand how each of these parameters changes the outcome of the process. A simulation allows the possibility of sacrificing reality to enhance other learning outcomes. Slow physical processes can be sped up, slowed down or even reversed to allow for a deeper understanding to be achieved. For students in the initial use phase, these deviations undermine their establishment of the reality of the simulation. For students in the regular and expert use phases, however, the reality is already established, and these deviations allow them to pursue the other learning objectives more effectively. 
Effectively, there are two different thresholds for adequate realism from a simulation - one that is sufficient for students to establish a sense of reality from the simulation, and a second, lower threshold that is necessary to maintain the sense of reality. These two thresholds correspond to the different learning objectives and contexts that students encounter during the different phases of their use.

\section{Conclusion}

Developing a simulation is a complex balancing act. There are many compromises that must be made to create the most effective learning environment within the constraints of the available resources. There is a general consensus that the more realistic the simulation, the better - but this consensus may be flawed.

The learning needs of students evolve throughout their interactions with simulations, with novice users having different learning objectives than experienced users. For a novice user, the belief in the fidelity of the simulation is an important learning outcome, so that they are willing to engage with the physical phenomena the simulation represents. While it is essential to establish that the simulation is an accurate representation of reality, it is not essential that this reality be maintained to the same level. Many of the features that are so impressive at first contact, and so useful in establishing the reality of a simulation, are in fact unnecessary bells and whistles for the experienced user.

The differences in the necessary levels of reality - the two distinct threshold model - offer new opportunities in the design and implementation of educational simulations. By focusing the resources into the areas that will provide the best return on investment, rather than into improving functionality that will ultimately go unused, educational simulations can be made more efficient and effective.

\section{References}

Aldrich, C., 2004. Simulations and the future of learning. San Francisco: Pfeiffer.

Alessi, S.M., and Trollip, S.R., 1991. Computer-based instruction: methods and development. 2nd ed. Englewood Cliffs, NJ: Prentice-Hall.

Ausubel, D.P., 2000. The acquisition and retention of knowledge: a cognitive view. Dordrect: Kluwer Academic.

Jia, R., et al., 2006. A virtual laboratory on fluid mechanics. American Society for Engineering Education, Chicago, IL.

Koretsky, M., et al., 2008. Enhancement of student learning in experimental design using a virtual laboratory. IEEE Transactions on Education, 51, 76-85.

Richardson, J.J., et al., 2006. Designing and implementing a virtual 3d microcontroller laboratory environment. Frontiers in education, San Diego.

Schofield, D., Lester, E., and Wilson, J.A., 2004. Virtual reality interactive learning environments. EE2004, Wolverhampton, UK, 225-231.

\section{About the authors}

Euan Lindsay is the Discipline Leader for Mechatronic Engineering at Curtin University of Technology, in Perth, Western Australia. His research interests include engineering education, telecontrol (particularly Internet-based telecontrol), artificial neural networks and rehabilitative technologies for people with sensing impairments. He is a member of the Executive of the Australasian Association for Engineering Education, and co-edits the Australasian Journal of Engineering Education. Dr Lindsay was the recipient of a 2007 Carrick Award for Australian University Teaching. In 2005, he was named as one of the 30 Most Inspirational Young Engineers in Australia. 
Steve Murray's professional background includes industrial computer systems development in Australia and the UK. He joined the UTS in 1993, having previously spent 6 years working on projects in industrial automation and flight simulation. After arriving in the Faculty of Engineering, he directed almost all of his efforts to the teaching and learning programmes. As well as developing and delivering a great number of undergraduate and postgraduate coursework subjects, and completing a term as Program Head of the Computer Systems Engineering and Software Engineering programmes, he has authored and co-authored several papers and a book chapter on topics related to remotely accessible laboratories. He was the team leader of a group which was honoured with a UTS Teaching Award in 2005 for work in this area, and received a 2006 Carrick Citation for work on remote laboratories.

Dikai Liu is an Associate Professor at the ARC Centre of Excellence for Autonomous Systems, Faculty of Engineering and Information Technology, University of Technology, Sydney. He has expertise in intelligent mechatronic systems. His research interests in engineering education include remote laboratory development, strategies to deploy remote laboratories in teaching and the effect of remote laboratories on student learning outcomes.

Professor David Lowe (BE PhD SMIEE, MACM) is the director of the Centre for Real-Time Information Networks (CRIN) in the Faculty of Engineering and Information Technology at the University of Technology, Sydney. He has active research interests in the areas of Web development and technologies, and software engineering. In particular he focuses on Web-based control and monitoring of distributed real-time systems. He has published widely, including three text books (most recently Web Engineering: A Practitioner's Approach, Roger Pressman and David Lowe, McGraw-Hill). He is also on numerous Web conference committees and journal editorial boards (including as a Managing Editor of the Journal of Web Engineering). He has undertaken numerous consultancies related to software evaluation, Web development (especially project planning and evaluation) and Web technologies. He is also passionate about teaching, and particularly the role of practice-based engineering education.

Chris Bright is a Research Associate in the Department of Mechanical Engineering at Curtin University of Technology. 